\title{
Synovial fluid proteome profile of surgical versus chemical induced osteoarthritis in rabbits
}

\author{
Sharifah Zakiah Syed Sulaiman ${ }^{1}$, Wei Miao Tan ${ }^{1}$, Rozanaliza Radzi $^{1}$, Intan Nur Fatiha Shafie ${ }^{1}$, Mokrish Ajat ${ }^{2}$,

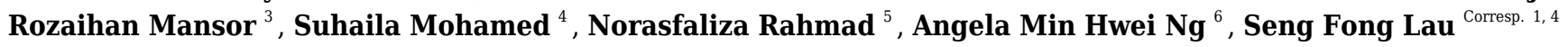 \\ 1 Department of Veterinary Clinical Studies, Universiti Putra Malaysia, Serdang, Selangor, Malaysia \\ Department of Veterinary Preclinical Studies, Universiti Putra Malaysia, Serdang, Selangor, Malaysia \\ 3 Department of Farm and Exotic Animals Medicine and Surgery, Universiti Putra Malaysia, Serdang, Selangor, Malaysia \\ Laboratory of Cancer Research UPM-MAKNA (CANRES), Institute of Bioscience, Universiti Putra Malaysia, Serdang, Selangor, Malaysia \\ 5 Agro Biotechnology Institute, Universiti Putra Malaysia, Serdang, Selangor, Malaysia \\ 6 \\ Tissue Engineering Centre, Universiti Kebangsaan Malaysia Medical Centre, Cheras, Selangor, Malaysia \\ Corresponding Author: Seng Fong Lau \\ Email address: lausengfong@upm.edu.my
}

Background: Animal models are significant for understanding human osteoarthritis (OA). This study compared the synovial fluid proteomics changes in surgical and chemical induced OA models.

Methods: Thirty rabbits either had anterior cruciate ligament transection (ACLT) procedure or injected intra-articularly with monosodium iodoacetate (MIA, $8 \mathrm{mg})$ into the right knee. The joints were anatomically assessed, and the synovial fluid proteins analyzed using two-dimensional polyacrylamide gel electrophoresis (2DGE) and MALDI TOF/TOF mass spectrometry analysis at 4, 8 and 12 weeks. The proteins' upregulation and downregulation were compared with control healthy knees.

Results: Seven proteins (histidine-rich glycoprotein, beta-actin-like protein 2 isoform X1, retinol-binding protein-4, alpha-1-antiproteinase, gelsolin isoform, serotransferrin, immunoglobulin kappa-b4 chain-Cregion) were significantly expressed by the surgical induction. They characterized cellular process (27\%), organization of cellular components or biogenesis (27\%), localization (27\%) and biological regulation (18\%), which related to synovitis, increased cellularity, and subsequently cartilage damage. Three proteins (apolipoprotein I-IV precursor, serpin peptidase inhibitor and haptoglobin precursor) were significantly modified by the chemical induction. They characterized stimulus responses (23\%), immune responses (15\%), biological regulations (15\%), metabolism (15\%), organization of cellular components or biogenesis $(8 \%)$, cellular process (8\%), biological adhesions (8\%) and localization (8\%), which related to chondrocytes glycolysis/death, neovascularization, subchondral bone necrosis/collapse and inflammation.

Conclusions: The surgical induced OA model showed a wider range of protein changes, which were most upregulated at week 12 . The biological process proteins expressions showed the chemical induced joints had slower OA progression compared to surgical induced joints. The chemical induced OA joints showed early inflammatory changes, which later decreased. 
1 SYNOVIAL FLUID PROTEOME PROFILE OF

2 SURGICAL VERSUS CHEMICAL INDUCED

3 OSTEOARTHRITIS IN RABBITS

$4 \quad{ }^{1}$ Sharifah Zakiah Syed Sulaiman, ${ }^{1}$ Wei Miao Tan, ${ }^{1}$ Rozanaliza Radzi, ${ }^{1}$ Intan Nur Fatiha Shafie, ${ }^{2}$ Mokrish

5 Ajat, ${ }^{3}$ Rozaihan Mansor, ${ }^{4}$ Suhaila Mohamed, ${ }^{5}$ Norasfaliza Rahmad, ${ }^{6}$ Angela Ng Min Hwei, ${ }^{1,4}$ Seng Fong

6 Lau

7

$8{ }^{1}$ Department of Veterinary Clinical Studies, Faculty of Veterinary Medicine,

9 Universiti Putra Malaysia, 43400, UPM Serdang, Selangor;

$10{ }^{2}$ Department of Veterinary Preclinical Studies, Faculty of Veterinary Medicine,

11 Universiti Putra Malaysia, 43400, UPM Serdang, Selangor;

$12{ }^{3}$ Department of Farm and Exotic Animals Medicine and Surgery, Faculty of Veterinary Medicine,

13 Universiti Putra Malaysia, 43400, UPM Serdang, Selangor;

$14{ }^{4}$ Laboratory of Cancer Research UPM-MAKNA (CANRES), Institute of Bioscience,

15 Universiti Putra Malaysia, 43400, UPM Serdang, Selangor;

$16{ }^{5}$ Agro Biotechnology Institute,

17 43400, Serdang, Selangor;

$18{ }^{6}$ Tissue Engineering Centre,

19 Universiti Kebangsaan Malaysia Medical Centre, 56000, Cheras, Kuala Lumpur.

20

21

22 Corresponding Author:

23 Seng Fong Lau ${ }^{1,4}$

24 Universiti Putra Malaysia, UPM Serdang, Selangor, 43400, Malaysia

25 Email address: lausengfong@hotmail.com; lausengfong@upm.edu.my

26

27

28

29

30

31

Peer] reviewing PDF | (2021:05:61310:2:0:NEW 28 Dec 2021) 


\section{Abstract}

33

34 Background: Animal models are significant for understanding human osteoarthritis (OA). This

35

36

37

38

39

40

41

42

43

44

45

46

47

48

49

50

51

52

53

54

55

56

57

58

59

60

61

62

63

study compared the synovial fluid proteomics changes in surgical and chemical induced OA models.

Methods: Thirty rabbits either had anterior cruciate ligament transection (ACLT) procedure or injected intra-articularly with monosodium iodoacetate (MIA, $8 \mathrm{mg}$ ) into the right knee. The joints were anatomically assessed, and the synovial fluid proteins analyzed using twodimensional polyacrylamide gel electrophoresis (2DGE) and MALDI TOF/TOF mass spectrometry analysis at 4, 8 and 12 weeks. The proteins' upregulation and downregulation were compared with control healthy knees.

Results: Seven proteins (histidine-rich glycoprotein, beta-actin-like protein 2 isoform X1, retinol-binding protein-4, alpha-1-antiproteinase, gelsolin isoform, serotransferrin, immunoglobulin kappa-b4 chain-C-region) were significantly expressed by the surgical induction. They characterized cellular process $(27 \%)$, organization of cellular components or biogenesis (27\%), localization (27\%) and biological regulation (18\%), which related to synovitis, increased cellularity, and subsequently cartilage damage. Three proteins (apolipoprotein I-IV precursor, serpin peptidase inhibitor and haptoglobin precursor) were significantly modified by the chemical induction. They characterized stimulus responses $(23 \%)$, immune responses (15\%), biological regulations (15\%), metabolism (15\%), organization of cellular components or biogenesis $(8 \%)$, cellular process $(8 \%)$, biological adhesions $(8 \%)$ and localization $(8 \%)$, which related to chondrocytes glycolysis/death, neovascularization, subchondral bone necrosis/collapse and inflammation.

Conclusions: The surgical induced OA model showed a wider range of protein changes, which were most upregulated at week 12. The biological process proteins expressions showed the chemical induced joints had slower OA progression compared to surgical induced joints. The chemical induced OA joints showed early inflammatory changes, which later decreased. 


\section{INTRODUCTION}

65

66

67

68

69

70

71

72

73

74

75

76

77

78

79

80

81

82

83

84

85

86

87

88

89

90

91

92

93

Osteoarthritis (OA) is a joint degeneration disease caused by the imbalance between cartilage degradation and synthesis, commonly due to aging. OA symptoms include joint pain, inflammation, morning stiffness, and decreased physical function, especially in the knee and hip. OA is characterized by deterioration of cartilage, formation of osteophyte and osteosclerosis, degradation of subchondral bone and tendons, inflammation of the synovium tissue, perforation of the trabecular plate, loss of trabecular bone and bone cysts (Burr \& Gallant, 2012; Li et al., 2013).

OA includes cartilage disorder and subchondral bone deterioration (Burr \& Gallant, 2012; Goldring \& Goldring, 2016). Articular cartilage degradation involves overexpression of matrix catabolic enzymes, inflammation, oxidative stress, and deterioration of proteoglycan and collagen matrix. Around $9.6 \%$ of males and $18 \%$ of females exceeding 60 years of age are impacted by OA (World Health Organization, 2013), resulting in reduced quality of life and productivity as well as an increased economic burden or medical cost (Salter et al., 2014). Current therapies, which include physiotherapy, orthopedic aids (orthoses), drugs, and surgery have limited efficacy. Pharmacological treatment has been limited due to adverse effects on cartilage (Huskisson et al., 1995) and gastrointestinal tract (Davies et al., 1997), whereas surgical intervention possesses many risks such as infection, blood clot and longer recovery rate.

Animal models used in pre-clinical OA research are classified into spontaneous and induced OA, further subcategorized into surgically or chemically induced OA models. Surgical OA induction modifies the joint strain/stress, leading to joint instability and damage. The anterior cruciate ligament transection (ACLT) is the most common method for surgical induced OA, entailing subchondral bone destruction and resulting in drastic cartilage changes (Lelovas, 2014). ACLT causes joint destabilization followed by post-traumatic osteoarthritis and reproduces the articular cartilage degradation after ACL injury (Piskin et al., 2007), or blunt trauma to cartilage, menisci, and subchondral bone (Teeple et al., 2013). The surgical model effectively yields a rapid OA progression; hence, it is convenient for short-term research but not for studies on early OA (Kuyinu et al., 2016). ACLT also causes changes in cell morphology and tissue equilibrium, especially in the femoral condyle cartilage (Buckwalter et al., 2005). For chemical induced OA, monosodium iodoacetate (MIA) is most commonly used (Guingamp et 
94 al., 1997; Takahashi et al., 2017). MIA suppresses the glyceraldehyde-3-dehydrogenase activity

95 required for glycolysis in chondrocytes, resulting in apoptosis, cartilage degradation, proteoglycan loss, functional joint impairment and subchondral bone lesions (Pitcher et al., 2016; Xie et al., 2012), similar to human OA.

Previous reports compared bone changes, histology, biochemistry and biomechanics of surgical versus chemical induced OA (Naveen et al., 2013) and concluded that chemical induced OA mimicked human OA. Each of the induced OA models has its own advantages, and no single model is considered the gold standard in assessing OA.

To the best of our knowledge, the synovial proteomic profiles between the two induced models have not been compared yet. This study analyses the proteome profiles of synovial fluids at different stages of surgical or chemical induced OA in rabbits, to provide further insights on the pathophysiology of OA and pave the road for future applications.

\section{METHODS}

\section{Animals}

Thirty male New Zealand white rabbits (A-Sapphire Enterprise, Malaysia) aged 8-9 months and weighing 1.8-2.0 kg were placed in Animal Research Facility, Faculty of Veterinary Medicine, Universiti Putra Malaysia. Each rabbit was kept in an individual stainless-steel cage at $22-25^{\circ} \mathrm{C}$, under a $12 \mathrm{hr} / 12 \mathrm{hr}$ light/dark cycle with $50-60 \%$ relative humidity. Rabbits were fed with commercial rabbit pellets (Penternakan Hong Lee Sdn. Bhd., Malaysia) and given fresh water ad libitum. The rabbits were acclimatized for one week prior to OA induction. Approval by the Institutional Animal Care and Use Committee (IACUC), Universiti Putra Malaysia was obtained for the experimental protocol (UPM/IACUC/AUP-R034). The rabbits were randomly grouped into surgically induced OA rabbits and chemically induced OA rabbits, with 15 individuals in each group. Rabbits were further subdivided into week 4, week 8 and week 12 time groups with 5 individuals in each group. OA was induced at the right knees of the animals, and the contralateral joint was used as a healthy control. 


\section{Preparation of animal model of surgically induced and chemically induced osteoarthritis}

123

124

125

126

127

128

129

130

131

132

133

134

135

136

137

138

139

140

141

142

143

144

145

146

147

148

149

150

Anterior cruciate ligament transection (ACLT) was done for surgically induced OA group on the right stifle with a scalpel, under surgically sterile conditions, as described by Vignon et al (1987). The animals were anesthetized with Zoletil® (Virbac, Australia) at 3mg/kg via intramuscular route and maintained with isoflurane (3\%) (Piramal Healthcare, India). In order to expose the anterior cruciate ligament, medial patella dislocation was performed by lateral parapatellar arthrotomy approach with the stifle joint being fully flexed. Once visualized, irrigation with sterile saline was made onto the stifle joint. Subsequently, using 4-0 polydioxanone suture, closure of the joint capsule and subcutaneous tissue was done. Skin closure was done using 3-0 nylon suture. Tramadol (Duopharma, Malaysia) 2mg/kg was given twice daily for three days as painkiller. The animals were caged with unrestricted movement until anaesthetic recovery.

The OA chemical induction was administered with $0.32 \mathrm{ml}$ of $25 \mathrm{mg} / \mathrm{ml}$ monosodium iodoacetate (MIA) (Sigma-Aldrich, USA) dissolved in saline, injected into the intraarticular space of the stifle joint, under general anaesthesia (Zoletil®) at $2 \mathrm{mg} / \mathrm{kg}$ via intramuscular route.

\section{Euthanization and sample collection}

Rabbits were visually inspected for any clinical signs, such as weight loss or immobility. In each group, rabbits were subdivided into week 4, week 8 and week 12 time groups. Because both joint amputations were required to collect the samples, rabbits were euthanized following the experiment with $120 \mathrm{mg} / \mathrm{kg}$ pentobarbital sodium (Vetoquinol, France) to avoid suffering caused by risk of infection and also immobilization. Knee joints were removed and immediately fixed in 10\% buffered formalin (Sigma-Aldrich, USA). After 24 hours, the muscle and tissue surrounding the femur and tibia were removed carefully to avoid damaging the cartilage surface. The proximal femur and distal tibia were placed in 10\% buffered formalin. Medial and lateral femoral condyle and tibial plateau of left and right stifle joints were photographed by P7000 digital camera (Nikon, Japan) at macro setting. They were examined for gross morphological changes, including erosions at the medial and lateral articular cartilage surfaces. Synovial fluids were collected via arthrocentesis, placed in a $1.5 \mathrm{ml}$ microcentrifuge tube and stored immediately at $-80^{\circ} \mathrm{C}$ until subsequent analysis.

Peer) reviewing PDF | (2021:05:61310:2:0:NEW 28 Dec 2021) 


\section{Histology}

152

153

154

155

156

157

158

159

160

161

162

163

164

165

166

167

168

169

170

171

172

173

174

175

176

177

178

179

180

181

Bones from harvested joint were decalcified immediately fixed in $10 \%$ buffered formalin (Sigma-Aldrich, USA) and were decalcified with 10\% formic acid (Nacalai Tesque, Japan) for 10 days. The femur and tibia were cut at a dorsal plane. Samples were dehydrated in a series of immersion in alcohol using Leica TP1020 Semi-enclosed Benchtop Tissue Processor (Leica Biosystem, Germany). Next, the samples were embedded in paraffin using Leica EG1150H and EG1 1559 Modular Tissue Embedding Center (Leica Biosystems, Germany) and sliced at $5 \mu \mathrm{m}$ using Reichert-Jung 2045 Multicut Rotary Microtome (Leica Biosystems, Germany) and mounted on glass slides. Prior to staining, the slides were deparaffinized and hydrated using distilled water. Next, they were stained using Fast Green (FCF) solution for five minutes and rinsed with $1 \%$ acetic acid before being stained using $0.1 \%$ Safranin-O solution for five minutes. The slides were dehydrated and cleared with 95\% ethyl alcohol and absolute ethyl alcohol, alternately at two minutes each for two times. The slides were then observed under microscope (Motic, China) under 20x magnification and scored independently by two blinded observers. The changes of articular cartilage were scored using OARSI Scoring System (Pritzker et al., 2006). Histological evaluation of subchondral bone was done by measuring the percentage of bone volume over tissue volume (BV/TV). The measurement was done according to Nagira et al. (2020). In brief, the compartments of osteochondral units were defined, including cancellous bone (Cn.B), bone marrow (BM) and trabecular bone (Tb.B)(Figure 1). The calculation of $\mathrm{BV} / \mathrm{TV}$ was done as follows: Bone volume $(\mathrm{BV} / \mathrm{TV}, \%)=(\mathrm{Cn} . \mathrm{B} \text { region }-\mathrm{BM} \text { regions })^{*} / \mathrm{Cn} . \mathrm{B}$ region $\times 100,(*$ Cn.B region $-\mathrm{BM}$ regions $=$ Tb.B regions $)($ Figure 1$)$. Calculations were done using ImageJ with bone histomorphometry method.

Figure 1: Definition of tissue compartment in subchondral bone. White square dotted line, cancellous bone (Cn.B), yellow round dotted line, bone marrow (BM). Calculation of BV/TV: Bone volume $(\mathrm{BV} / \mathrm{TV}, \%)=($ Cn.B region $-\mathrm{BM}$ regions $) * / \mathrm{Cn} . \mathrm{B}$ region $\times 100,{ }^{*} \mathrm{Cn} . \mathrm{B}$ region $-\mathrm{BM}$ regions $=\mathrm{Tb} . \mathrm{B}$ regions.

\section{Statistical analysis}

Sample size estimation using power analysis was performed before conducting the experiment, which was based on prior work that detected 23 significantly differentially 
182 expressed proteins between OA induction group and control group (Luo et al., 2018). A sample 183 size of seven rabbits per group were determined $(\alpha=0.05$, power $>80 \%)$. However, in view of 184 the large sample size, sample size of 5 rabbits per group was chosen as suggested by the Animal 185 Research Committee. Power analysis was done using G*Power software (Faul et al., 2007). 186 Articular cartilage scoring data were tabulated as median and statistical comparisons for scoring 187 between groups were analysed using the Kruskal-Wallis (K-W) non-parametric ANOVA and 188 followed up with Dunn's multiple comparison tests. BV/TV value of subchondral bone 189 evaluation were analysed using one-factor analysis of variance (ANOVA) and multiple 190 comparison among groups were analysed using Tukey's HSD post hoc test. All analysis were 191 done using Graphpad Prism 7 (Graphpad Software, USA). The level of significance was at $192 \alpha=0.05$.

\section{Protein precipitation from synovial fluid}

Proteins from synovial fluid were precipitated using modified TCA/Acetone precipitation method (Chen et al., 2005). Before protein precipitation, synovial fluid samples were centrifuged at $3000 \mathrm{x} \mathrm{g}$ at ambient temperature for 10 minutes to remove cells and cellular debris. The synovial fluid samples were pooled for each group and precipitated with cold $10 \%$ trichloroacetic acid (TCA) (Chemiz, Malaysia) in 1:4 ratio inside a microcentrifuge tube. Samples were kept for 60 minutes in a $-20{ }^{\circ} \mathrm{C}$ chiller, before centrifugation at $12000 \mathrm{x}$ g for 60 minutes at $4{ }^{\circ} \mathrm{C}$. The supernatant was removed without disrupting the protein pellet. Protein pellets were washed three times with cold $100 \%$ acetone and centrifuged at $14000 \mathrm{x}$ g for 20 minutes, then the supernatant was removed. Finally, the microcentrifuge tube was inverted on the C-fold tissue and air-dried for 30 seconds.

204

205

206

207

208

209

210

\section{Protein concentration estimation}

Protein pellets were resuspended and solubilized with rehydration buffer containing $7 \mathrm{M}$ urea, $2 \mathrm{M}$ thiourea, $4 \% \mathrm{CHAPS}$, and $0.002 \%$ bromophenol blue. Protein concentration was determined using the 2D Quant Kit (GE Healthcare, Sweden), following manufacturer's protocol. A standard curve was obtained using $2 \mathrm{mg} / \mathrm{ml}$ Bovine serum albumin (BSA) stock solution. 500 $\mu 1$ precipitant was inserted into each tube, vortexed and incubated at ambient temperature for 2-3 minutes, then $500 \mu 1$ co-precipitant was added. Tubes were then vortexed and centrifuged for 5 
211 minutes at $10000 \mathrm{x}$ g and supernatant discarded. The tubes were centrifuged again. The residual 212 supernatant was decanted. Copper solution $(100 \mu \mathrm{l})$ and distilled water $(400 \mu \mathrm{l})$ were added to 213 each tube, and precipitated protein was dissolved by vortexing. The working reagent (1 ml) was 214 added to each tube and mixed by inversion instantaneously. Absorbance was measured using 215 Cary®50 UV-Vis spectrometer at $480 \mathrm{~nm}$ (Varian Inc., United States).

216

217

218

219

220

221

222

223

224

225

226

227

228

229

230

231

232

233

234

235

236

237

238

239

\section{Isoelectric focusing}

Synovial fluid protein $(250 \mu \mathrm{g})$ was rehydrated overnight using $13 \mathrm{~cm}$ immobilized $\mathrm{pH}$ gradient strips pH 3-10 ImmobilineTM DryStrip gels (IPG) (GE Healthcare, Sweden). The samples were focused with a low starting voltage followed by a voltage gradient of 15000 to 20 $000 \mathrm{~V}$, with a $50 \mathrm{~mA}$ strip limiting current. Strips were stored at $-80^{\circ} \mathrm{C}$ until further analysis.

\section{Two-dimensional gel electrophoresis (2DGE)}

For 2DGE, strips were first equilibrated for 15 minutes with $50 \mathrm{mg}$ dithiothreitol (DTT) in equilibrium solution (50 mM tris- $\mathrm{HCl} \mathrm{pH}$ 8.8, $6 \mathrm{M}$ urea, $30 \%$ glycerol, $2 \% \mathrm{SDS}, 0.002 \%$ bromophenol blue) (GE Healthcare, Sweden), followed by 15 minutes alkylation using $125 \mathrm{mg}$ iodoacetamide (IAA) (GE Healthcare, Sweden) in equilibrium solution. The strips were positioned on top of $12 \%$ gradient slab polyacrylamide gels and secured with molten $0.5 \%$ agarose in running buffer ( $25 \mathrm{mM}$ tris $\mathrm{pH} 8.3 ; 198 \mathrm{mM}$ glycine; 0.1 percent w/v SDS) with a drop of bromophenol blue. Gels were run at $140 \mathrm{~V}$ for 10 minutes, then at $200 \mathrm{~V}$ for 55-60 minutes using Ruby SE 600 system (GE Healthcare, Sweden). Gels were stained using colloidal Coomassie R350 (GE Healthcare, Sweden) and destained using destaining solution (40\% methanol, $7 \%$ glacial acetic acid, $53 \%$ distilled water).

Gel images were obtained using a GS-800TM Optimized Densitometer for intra-sample and inter-sample variability evaluation (Bio-Rad Laboratories, US). The digitized images were aligned and analyzed using Progenesis SameSpots software (Nonlinear Dynamics, Durham, NC). According to manufacturer's instruction, spots were detected, matched and normalized. Differential protein spots between control and induced models were determined using normalized spots and compared with reference gel image. Fold difference and p-values were calculated using one-way ANOVA. Threshold value was set at 1.5 fold change for upregulation and downregulation with $\mathrm{p} \leq 0.05$. 
240

241

242

243

244

245

246

247

248

249

250

251

252

253

254

255

256

257

258

259

260

261

262

263

264

265

266

267

268

269

\section{Protein digestion and matrix-assisted laser desorption/ionization time of flight/time of flight mass spectrometry (MALDI-TOF/TOF)}

Gels were rinsed twice using distilled water, and 2DGE spots were excised manually under sterile conditions. Spots were digested in a two-day process. On day one, the excised gel spots were washed with $150 \mu \mathrm{l}$ of $100 \mathrm{mM}$ ammonium bicarbonate (ABC) for 10 minutes and vortexed. Following that, wash solution ( $50 \%$ acetonitrile in $100 \mathrm{mM} \mathrm{ABC})$ was added twice, 5 minutes for each wash. This process was repeated until the gel dye was utterly destained. Reduction solution (150 $\mu \mathrm{l}$ of $10 \mathrm{mM}$ DTT in $100 \mathrm{mM} \mathrm{ABC})$ was then added and gel spots were incubated at $60{ }^{\circ} \mathrm{C}$ in water bath for 30 minutes. Alkylation solution $(150 \mu \mathrm{l}$ of $55 \mathrm{mM}$ IAA in $100 \mathrm{mM}$ ABC) was added before incubation in the dark for 20 minutes. The solution was removed, and wash solution was added twice, 20 minutes for each wash. Next, $50 \mu 1$ of $100 \%$ acetonitrile was added and incubated at ambient temperature for 15 minutes. Gel spots were then vacuum-dried using ScanVac (Labogene, Denmark) for 1 hour. Finally, $25 \mu 1$ of $7 \mathrm{ng} / \mu \mathrm{l}$ trypsin solution were added, before incubation overnight at $30{ }^{\circ} \mathrm{C}$. On the next day, $25 \mu 1$ of $50 \%$ acetonitrile were added and gel spots were incubated for 15 minutes at ambient temperature. After centrifugation at $14000 \mathrm{xg}$, supernatant samples were collected and transferred to new tubes. Then, $20 \mu \mathrm{l}$ of $100 \%$ acetonitrile were added before incubation for 15 minutes. The obtained solution was transferred into a new tube and the peptide was vacuum-dried and resuspended with $5 \mu \mathrm{l}-10 \mu \mathrm{l}$ of $0.1 \%$ trifluoroacetic acid. The digested protein sample underwent desalting and concentration using $\mu$ ZipTipC18 pipette tips (Millipore, Bedford, MA, USA). Protein samples were then analyzed using ultraflextreme MALDI-TOF/TOF mass spectrometer (Bruker, Bremen, Germany). Peptide-matrix compounds were loaded using the dried droplet technique on the instrument target plate. The used matrix compound was $\alpha$-cyano-4hydroxycinnamic acid (CHCA) (Bruker, Bremen, Germany). Samples were analyzed with MASCOT version 3.5 (Matrix science, USA) using the MASCOT search engine against the sequence of Oryctolagus cuniculus species in the NCBInr database. Samples with $\mathrm{p} \leq 0.05$ were accepted as significant.

\section{RESULTS}

\section{General condition of rabbit models}



surgically induced OA joints showed a more severe time-dependent swelling and warmth. Both 272 groups showed no clinical signs of pain, immobility or reduction in body weight and were

273 healthy prior to euthanization. The rabbits' average weight prior to euthanasia is $2.4 \mathrm{~kg}$ and 274 ranged from $1.8 \mathrm{~kg}-3.6 \mathrm{~kg}$.

275

276

277

278

279

280

281

282

283

284

285

286

287

288

289

290

291

292

293

294

295

296

297

298

\section{Visual inspection of the joint and gross anatomy of the femoral condyle and tibial plateau}

The gross morphology of the femoral condyle and tibial plateau showed no significant changes before week 4 and were similar to the control. The articular cartilage of the joints from both induced OA groups was smooth and polished without any surface irregularities. OA features such as surface erosions were observed on the femur and the tibia of both groups in week 8. Erosions were more notable on the femur lateral condyle cartilage but had generalized distribution on the tibia articular cartilage. The joints displayed similar significant osteoarthritic features in both induced OA groups at week 12. Joints from surgically induced OA had more apparent erosion on the medial condyle of femoral and tibial cartilages. In contrast, joints from chemically induced OA showed evenly distributed erosions at the articular cartilage surface.

\section{Histopathological articular cartilage grading, subchondral bone evaluation and statistical} analysis

Significant cartilage erosion was found in surgically induced and chemically induced OA group whereas control group showed a normal chondrocytes distribution and smooth cartilage surface in both femur and tibia. OARSI histopathology score was shown in Table 1 and Figure 2, and representative histological images for femur and tibia was shown in Figure 3 and Figure 4. For femur, in the surgically induced group, uneven articular surface and superficial fibrillation were observed (Fig. 3B, 3C) at week 4 and week 8 after induction. Superficial zone fibrillation was observed during week 12. Chemically induced group showed a more severe articular cartilage changes indicated by cartilage erosion which was observed during week 8 and week 12 (Fig. 3G, 3H). For tibia, surgically induced group showed superficial fibrillation and chondrocytes apoptosis indicated by empty chondrons during week 8 . During week 12, deep fibrillation and surface discontinuity was observed. Again, chemically induced group showed a more severe changes with surface erosion presence during week 8 and week 12 (Fig. 4G, 4H). 
Histopathological evaluation of subchondral bone recorded decreased BV/TV value in

300

301

302

303

304

305

306

307

308

309

310

311

312

313

314

315

316

317

318

319

320

321

322

323

324

325

326

327

328

329

330

surgically induced group for week 4 and week 8 in comparison with control (Fig. 5). At week 12, $\mathrm{BV} / \mathrm{TV}$ value in surgically induced group increased slightly compared to week 8 . For chemically induced group, BV/TV value decreased persistently from week 4 until week 12. The same pattern was recorded for both induction groups for tibia bone.

Table 1: OARSI histopathological grade of femur and tibia in the control, week 4, week 8 and week 12 for the surgically and chemically induced group

Figure 2: OARSI histopathological scores for (A) surgically induced femoral cartilage (B) chemically induced femoral cartilage (C) surgically induced tibial cartilage (D) chemically induced tibial cartilage. Values are presented in median with $95 \%$ confidence interval $(n=5)$. Significant difference (Dunn's multiple comparisons, $\mathrm{p}<0.05$ ) between column with the same letter within the groups.

Figure 3: Representative histology images of femoral articular cartilage. A-D: surgically induced group. E-H: chemically induced group. Safranin-O/fast green stained with 20x magnification.

Surgically induced group showed apoptosis indicated by empty chondrons (red arrow) during week 4 and superficial fibrillation (red asterisk) during week 8. Chemically induced group showed surface erosion as early as week 8 (red arrowhead).

Figure 4: Representative histology images of tibial articular cartilage A-D: surgically induced group. E-H: chemically induced group. Safranin-O/fast green stained with 20x magnification. During week 4, surgically induced group showed chondrocyte apoptosis (red arrow) and fibrillation (red asterisk) during week 8. Cartilage erosion (red arrowhead) were present during week 12. For chemically induced group, erosion can be observed during week 8 and week 12 .

Figure 5: Subchondral bone BV/TV values for (A) femur and (B) tibia in week 4, week 8 and week 12 for the control, surgically (ACLT) and chemically (MIA) induced group. No significant difference between groups were reported. The results are represented as mean $( \pm \mathrm{SD})$.

\section{Evaluation of protein yield and two-dimensional gel electrophoresis analysis}


The precipitated protein concentrations from both induction groups ranged between 3.11-

332

333

334

335

336

337

338

339

340

341

342

343

344

345

346

347

348

349

350

351

352

353

354

355

356

357

358

359

360

$19.9 \mu \mathrm{g} / \mathrm{ml}$. The 2DGE gels showed that throughout OA progression protein expression profiles are different from healthy control. The joints from surgically-induced OA displayed 28 significant matched spots ( $\leq \leq 0.05$; fold change expression $\geq 1.5$ ) (Fig. 6A), whereas joints from chemical induced OA displayed a total of 23 matched protein spots $(\mathrm{p} \leq 0.05$; fold change expression $\geq 1.5$ ) (Fig. 6B). The log normalized volume were calculated using SameSpot software. The spots were then manually excised and identified using MALDI-TOF/TOF.

Figure 6: Representative 2DGE (pI 3-10) image for rabbit synovial fluid in (A) surgically induced group and (B) chemically induced group after staining with colloidal Coomassie stain. Gels from respective groups were scanned and analyzed with Progenesis SameSpot software and protein spots (circled in red) were selected to be picked.

\section{Identification of proteins spots}

Twenty eight and 11 non-redundant proteins were identified from joints with surgical induced and chemical induced OA, respectively. However, only seven proteins from surgical induced OA joints and three proteins from the chemical induced OA joints have significantly different ( $\mathrm{p} \leq 0.05$ ) fold-change expressions $(\geq 1.5)$ when analyzed as log normalized volume (Table 2).

Table 2: List of proteins and their log normalized volume for control, week 4, week 8 and week 12 for both induction groups. The results are represented as mean $( \pm \mathrm{SD})$.

The proteins were classified by their biological process functions (Table 3). In the surgically induced OA joints, proteins were characteristics of cellular process $(27 \%)$, organization of cellular components or biogenesis (27\%), localization (27\%) and biological regulation (18\%). For chemically induced OA joints, proteins were involved in stimulus responses (23\%), immune responses (15\%), biological regulations (15\%), metabolism (15\%), organization of cellular components or biogenesis $(8 \%)$, cellular process $(8 \%)$, biological adhesions $(8 \%)$ and localization $(8 \%)$.

Table 3: Characterization of proteins according to biological group for surgically and chemically induced group. 
361

362

363

364

365

366

367

368

369

370

371

372

373

374

375

376

377

378

379

380

381

382

383

384

385

386

387

388

389

\section{DISCUSSION}

The effusion developments in the surgically induced OA joints were observed as early as week 4 and increased significantly until week 12. Other reports on in vivo OA rabbit models detected severe joint effusion 2 weeks post-surgery, which grew prominent at week 8 . The effusion in the surgically induced OA joints was more severe compared to the chemically induced OA joints, probably indicating greater post-operative inflammation severity (Bouchgua et al., 2009).

The gross morphology suggested that the OA damage in both groups was only manifest after 8 weeks post-induction. In surgically induced OA, joint lesions were more prominent on the medial condyle area, which is similar in humans, where the medial compartment is the most commonly affected. This relates to the meniscus functions, as the medial meniscus is the more important attachment for tibia movement at the knee (Campos et al., 2013). Thus, the ACLT model most effectively mimics human post-traumatic osteoarthritis (PTOA). Histological assessment of cartilage and subchondral bone is accepted as a gold standard in assessing and monitoring OA progression in animal models. This assessment can be used to observe different stages of joint degeneration (Rutgers et al., 2010).

Histological analysis revealed slight articular cartilage changes in surgically and chemically induced (Man \& Mologhianu, 2014) OA during week 4 which indicated initiation of OA in both groups that may be pronounced over time. This is in line with previous report in which ACLT model in rabbits showed slower lesion progression between 3 and 6 weeks and the intensity of the lesion increases 6 weeks post-induction (Campos et al., 2013). During week 8 and week 12, more severe changes were observed in chemically induced group based on higher histological scoring. Similarly, previous study detected obvious degenerative changes in the cartilage as early as six to ten weeks after monosodium iodoacetate induction (Mohan et al., 2011). Subchondral bone histopathological evaluation in surgically induced group showed increased BV/TV values during week 12, suggesting the occurrence of bone remodeling indicating higher OA progression (Fell et al., 2019). As for chemically induced group, continuous decline in BV/TV throughout 12 weeks may be due to less loading of induced knee which triggered weight distribution alterations (Guingamp et al., 1997). 
390

391

392

393

394

395

396

397

398

399

400

401

402

403

404

405

406

407

408

409

410

411

412

413

414

415

416

417

418

Induction using monosodium iodoacetate will lead to chondrocytes apoptosis that will first cause changes in morphological and histological features of articular cartilage before changes in subchondral bones (Naveen et al., 2013). On the other hand, surgical induction using ACLT method will cause destabilization of the joint and increased load at subchondral bone. Osteoclasts' activity will increase and induced subchondral bone loss (Botter et al., 2009) and subsequently bone remodeling at later stages. Therefore, subchondral bone resorption is the initiating factor for articular cartilage degradation in surgically induced group and it can be concluded that changes in the bone preceded changes in the articular cartilage in ACLT induction (Yang et al., 2020).

The proteome profiles revealed four similar proteins detected in both groups: histidinerich glycoprotein (HRG), serotransferrin, alpha-1-antiproteinase and immunoglobulin. Only HRG, serotransferin and alpha-1-antiproteinase expressions were significantly changed in the surgically induced OA joints. Immunoglobulin is one of the most highly expressed proteins, and its presence in both groups was expected since no depletion kit was used in this study. HRG, detected in both groups, is a multifunctional plasma protein interacting with the immune system. Previous studies showed that HRG binds strongly to some complementary proteins such as c4b binding protein, $\mathrm{C} 1 \mathrm{q}$, and factor $\mathrm{H}$, and is one of the most present proteins in rheumatoid arthritis patients' synovial fluid and plasma (Manderson et al., 2009). However, the role of HRG in OA is still unclear.

An upregulation of most identified proteins was observed in joints from surgically and chemically induced OA. Nevertheless, surgically induced OA displayed slight downregulation of alpha-1-antiproteinase protein, an acute-phase protein that protects tissue from uncontrolled proteolysis damage and is upregulated by pro-inflammatory interleukin-6 (Ucar et al., 2007). Such decrease indicates that inflammation and chondroprotection loss started at week 4 in the surgically induced OA model.

The retinol-binding protein 4 (RBP-4) was more upregulated in week 4 compared to week 8 and week 12. It belongs to the lipocalin family and is a blood retinol carrier positively correlated with the collagen degrading matrix metalloproteinases (MMPs) MMP-1 and MMP-13 (Scotece et al., 2018). MMP-13 was released in response to mechanical injury or joint 
419 destabilization (Rose \& Kooyman, 2016), so this could explain RBP-4 expression in surgically 420 induced OA joints.

421 In chemically induced OA joints, apolipoprotein I-IV precursor and serpin peptidase 422 inhibitor proteins were most upregulated at week 4 compared to week 8 and week 12 . 423 Apolipoprotein is a pro-inflammatory acute-phase protein involved in lipid and cholesterol 424 metabolism, found to be upregulated synovial fluids of OA patients (Nguyen et al., 2017). 425 Apolipoprotein induces IL-6 expressions in human primary chondrocytes (De Seny et al., 2015). 426 Serpin peptidase inhibitor inhibits serine proteases and MMPs regulation, aggrecanase plasmin, 427 angiogenesis activity, tissue mitogens and inflammatory leukocyte proteases (Gobezie et al., 428 2007) in OA. These protein control processes such as inflammation and coagulation. The 429 upregulation of apolipoprotein suggested that joint inflammation occurs early at week 4 post430 induction. This consequently caused increased serpin peptidase inhibitor expression, which is 431 negative feedback for MMP regulation following OA-related joint inflammation. observed in surgical induced OA joints compared to the control group. Upregulation during week 4 followed by downregulation during week 8 indicated that OA progression was ongoing. Betaactin-like protein 2 isoform $\mathrm{X} 1$ is a highly conserved protein responsible for cell motility, structure and integrity. Beta-actin-like protein 2 isoform X1 was most upregulated in the femur lateral secretome of early OA patients (Stenberg et al., 2013) and was also identified in synovial fluid of OA patients (Gobezie et al., 2007).

In chemically induced OA joints, the haptoglobin precursor was highly upregulated.

440 Haptoglobin has immunomodulatory effects and reduces hemolysis-associated oxidative damage 441 by binding with free hemoglobin (Cray et al., 2009). The upregulation of haptoglobin precursor 442 indicated that OA progressed from week 4 and persisted until week 8. A previous study reported 443 haptoglobin expression was reduced as much as 70\% in early shoulder OA patients (Wanner et 444 al., 2013). The increased haptoglobin level in advanced OA is due to later upstream cytokine 445 release (Liao et al., 2015).

446 In the surgically induced OA joints, the strongest upregulation of gelsolin and 447 serotransferrin occurs at week 12, indicating advanced stage OA. Gelsolin is a major 448 extracellular actin scavenging system component and a novel MMP-14 substrate, as shown in a 
449 previous study that demonstrates the cleavage of plasma gelsolin by MMP-14 (Park et al., 2006).

450 MMP-14 can activate MMP-2 and MMP-13, involved in tissue turnover and upregulated in

451 diseased state (Rose \& Kooyman, 2016). Gelsolin was decreased in rheumatoid arthritis patients

452 (Osborn et al., 2008) but increased in OA individuals (Mateos et al., 2012), where it is

453 considered a putative biomarker. Thus, gelsolin upregulation in surgically induced OA joints

454 from the early stage until week 12 indicates rapid disease progression because of MMP-14

455 activation.

456

Serotransferin upregulation was revealed in both types of OA joints. Serotransferin is

457 secreted from the liver and involved in iron transport from absorption sites, from heme

458 degradation to storage and utilization (Nylund et al., 2014). Serotransferin is a traditional acute-

459 phase protein that reflects increased OA and RA inflammatory conditions. In this study,

460 serotransferin levels are slightly downregulated in chemically induced joints and upregulated in

461 surgically induced joints. Other studies reported serotransferin downregulation in OA (Gharbi et

462 al., 2011; Nylund et al., 2014), which contradicts the results from our surgically induced model.

463 Serotransferrin was downregulated in early shoulder OA patients but upregulated by $50 \%$ in late

464 OA patients (Wanner et al., 2013). This suggests that based on the protein expressed, surgically

465 induced OA progression may be more advanced at week 12 compared to chemically induced

466 OA.

467 Overall, surgically induced OA development occurred immediately after induction and

468 continuously progressed until the end of the experimental duration (week 12), when most of the

469 identified proteins were strongly upregulated. In chemically induced OA joints, the levels of the

470 identified proteins were highest during the initial stage of induction and lowered at the final

471 stage, suggesting that chemically induced OA develops rapidly during the initial stage but slows

472 down or partially recovers at the later stages, based on the results at the molecular level. In

473 surgically induced group, destabilization of the joint will trigger the release of proinflammatory

474 cytokines which subsequently activates acute phase response that were expressed by alpha-1-

475 antiproteinase protein. Concurrently, retinol binding protein-4 and gelsolin release activated

476 MMP related pathways. As for chemically induced group, increased articular cartilage

477 degradation will continue to release pro-inflammatory cytokines which in turn stimulated the

478 release of acute phase proteins such as haptoglobin precursor and apolipoprotein I-IV precursor 
479 which involved in acute phase response. In addition, local response in the joint triggered a 480 negative feedback mechanism on the inflammatory response through the expression of serpin 481 peptidase inhibitor.

$482 \quad$ Surgically induced OA joints were associated with more cellular process changes.

483 Synovitis is characterized by increased cellularity and cartilage damage at the advanced OA 484 stages. Surgically induced OA mimics post-traumatic OA, which promotes angiogenesis (Liu et 485 al., 2019). In surgically induced OA joints, angiogenesis involves cellular biological migration, 486 growth and differentiation of endothelial cells, and organization of cellular components.

487 Apolipoprotein is the metalloproteinase substrate that modulates angiogenic responses

488 (Quintero-Fabián et al., 2019). Gelsolin, which is upregulated in surgically induced OA joints, is 489 an important regulator of cellular functions for osteoclasts motility and podosomes (cell adhesion 490 structures) formation (Silacci et al., 2004).

491

492

493

494

495

496

497

498

499

500

501

502

503

504

505

506

507

508

Chemically induced OA joints were associated with more diverse biological processes, although this group has fewer significant protein changes. The most represented proteins were involved in response to stimulus (23\%) and in immune responses, biological regulation, and metabolic process (15\%). The MIA induction disrupts chondrocytes glycolysis by suppressing glyceraldehyde-3-phosphatase dehydrogenase, subsequently causing mitochondrial pathway chondrocyte apoptosis involving reactive oxygen species (ROS) production (Jiang et al., 2013), neovascularization, necrosis and collapse of subchondral bone, as well as inflammation (Pitcher et al., 2016). These processes involve changes in cell states and activities following a stimulus, thus causing major upregulation of stimulus-response proteins. In chemical induced OA joints, serpin peptidase inhibitor upregulation inactivates proteinase inhibition by ROS-associated oxidative inactivation, which causes further joint inflammation (Jones et al., 1998). Haptoglobin precursor and apolipoprotein are acute-phase proteins, released or activated in acute response following defective chondrocyte metabolism (Sipe, 1995). In auto-inflammatory and non-autoinflammatory OA, immune regulation may be crucial for altering cell osmosis and inflammatory response in periostitis, which is associated with osteophytes formation in OA (Lu et al., 2014).

This study indicates that surgically induced OA developed more rapidly compared to chemically induced OA, based on the expressed biological process of proteins associated with advanced stage OA. The choice of OA induction is depending on the type of study that will be 
509 conducted, where surgically induced OA is suitable studies with a shorter time frame and

510 chemically induced OA is suited for studies that needed a longer duration.

511 Several limitations were noted in this study. The main limitation to this study is the small

512 numbers of animals in each group $(n=5)$ for each time point which also reflected the absence of

513 sham control group. This is due to suggestion by Animal Care Committee as a consideration for

514 the welfare of the rabbits. While this may hampers a more robust conclusion, it does provide

515 significant preliminary data which may justify larger scale studies to be conducted. Also,

516 decision to use contralateral joint as control was to reduce the number of animals to be

517 sacrificed. In a study by Mustafy et al. (2018), contralateral joint can be accepted as a suitable

518 control model as they showed high degree of similarities, with no significant differences among

519 all evaluated mechanical, geometrical and morphological parameters. In addition, proteomics

520 analysis was done using synovial fluid which was in direct contact with primary tissues of each

521 joint. Hence, the results obtained for each joint would not completely correlated with each other

522 as proteins expressed in each joint were relatively different as synovial fluid reflected the

523 condition of each individual joint, showed focal changes in particular joints and therefore was

524 reliable in characterizing changes in control and experimental joints.

525 The limitations in using rabbit models is the difference in joint biomechanics and gait in 526 comparison with human, and structural difference in joint tissues (McCoy, 2015). However,

527 although the translatability of each animal models to human clinical conditions are different,

528 rabbit models are one of the widely used animal models as it is easily reproducible, low cost and 529 easier in handling.

530 Changes in meniscus and synovium can also be observed to further elucidate disease

531 progression as OA will cause degenerative changes in the meniscus by the loss of Type 1 and

532 Type II collagen. In addition, synovial macrophage which is originated from synovium will be

533 activated during inflammation process (Kuyinu et al., 2016). However, in this study, as synovial

534 fluid is originated from synovium that releases various cytokines and pro-inflammatory

535 mediators during disease progression (Danila, 2014), we would like to propose a non-invasive or

536 less invasive method to assess the progression of OA using synovial fluid. This method will

537 provide an early indication of OA development and intervention to stop or reduce further

538 degeneration of joint and can represent OA progression. 
539

540

541

542

543

544

545

546

547

548

549

550

551

552

553

554

555

556

557

558

559

560

561

562

563

564

\section{CONCLUSIONS}

This study showed the surgical induced model showed a wider range of proteome profile and had the highest upregulation of most proteins at week 12. On the other hand, chemically induced joints have slower OA progression compared to surgically induced joints, based on the biological process proteins expressed. The chemically induced joints showed inflammatory changes at the early phase but had decreased expression at the later stages.

\section{ACKNOWLEDGEMENTS}

We would like to thank Dr. Murshidah Mohd Asri and Dr. Goh Soon Heng for helping during the study.

\section{REFERENCES}

Botter SM, Glasson SS, Hopkins B, Clockaerts S, Weinans H, van Leeuwen JPTM, van Osch GJVM. 2009. ADAMTS5-/- mice have less subchondral bone changes after induction of osteoarthritis through surgical instability: implications for a link between cartilage and subchondral bone changes. Osteoarthritis and Cartilage. DOI:

10.1016/j.joca.2008.09.018.

Bouchgua, M., Alexander, K., André d'Anjou, M., Girard, C. A., Carmel, E. N., Beauchamp, G., Richard, H., \& Laverty, S. (2009). Use of routine clinical multimodality imaging in a rabbit model of osteoarthritis - part I. Osteoarthritis and Cartilage. https://doi.org/10.1016/j.joca.2008.06.017

Buckwalter, J. A., Mankin, H. J., \& Grodzinsky, A. J. (2005). Articular cartilage and osteoarthritis. In Instructional course lectures. https://doi.org/10.1136/ard.51.9.1028-a

Burr DB, Gallant MA (2012) Bone remodelling in osteoarthritis. Nature Reviews Rheumatology.

Campos, W. N. S., Souza, M. A., Ruiz, T., Peres, T. P., Néspoli, P. B., Marques, A. T. C., Colodel, E. M., Souza, R. L. De, S, A. C. W. N., Souza, M. A., Ruiz, T., Peres, T. P., Néspoli, P. B., \& Marques, A. T. C. (2013). Experimental osteoarthritis in rabbits : lesion progression 1. 32(3), 279-285.

Peer] reviewing PDF | (2021:05:61310:2:0:NEW 28 Dec 2021) 
565 Chen, Y. Y., Lin, S. Y., Yeh, Y. Y., Hsiao, H. H., Wu, C. Y., Chen, S. T., \& Wang, A. H. J.

566

567

568

569

570

571

572

573

574

575

576

577

578

579

580

581

582

583

584

585

586

587

588

589

590

(2005). A modified protein precipitation procedure for efficient removal of albumin from serum. Electrophoresis. https://doi.org/10.1002/elps.200410381

Cray, C., Zaias, J., \& Altman, N. H. (2009). Acute phase response in animals: A review. In Comparative Medicine.

Danila MI. 2014. Biology of Normal Joint and Evaluation of the Joint Including CLinical, Imaging, and Pathologic Evaluation. In: Pathobiology of Human Disease: A Dynamic Encyclopedia of Disease Mechanisms. DOI: 10.1016/B978-0-12-386456-7.04301-X.

Davies, N. M., Roøseth, A. G., Appleyard, C. B., Mcknight, W., Del Soldato, P., Calignano, A., Cirino, G., \& Wallace, J. L. (1997). NO-naproxen vs. naproxen: Ulcerogenic, analgesic and anti-inflammatory effects. Alimentary Pharmacology and Therapeutics. https://doi.org/10.1046/j.1365-2036.1997.115286000.x

De Seny, D., Cobraiville, G., Charlier, E., Neuville, S., Lutteri, L., Goff, C. Le, Malaise, D., Malaise, O., Chapelle, J. P., Relic, B., \& Malaise, M. G. (2015). Apolipoprotein-A1 as a damage-associated molecular patterns protein in osteoarthritis: Ex vivo and in vitro proinflammatory properties. PLoS ONE. https://doi.org/10.1371/journal.pone.0122904

Faul F, Erdfelder E, Lang AG, Buchner A. 2007. G*Power 3: A flexible statistical power analysis program for the social, behavioral, and biomedical sciences. In: Behavior Research Methods. DOI: 10.3758/BF03193146.

Fell NLA, Lawless BM, Cox SC, Cooke ME, Eisenstein NM, Shepherd DET, Espino DM. 2019. The role of subchondral bone, and its histomorphology, on the dynamic viscoelasticity of cartilage, bone and osteochondral cores. Osteoarthritis and Cartilage. DOI: 10.1016/j.joca.2018.12.006.

Gharbi, M., Deberg, M., \& Henrotin, Y. (2011). Application for proteomic techniques in studying osteoarthritis: A review. Frontiers in Physiology. https://doi.org/10.3389/fphys.2011.00090

Peer] reviewing PDF | (2021:05:61310:2:0:NEW 28 Dec 2021) 
591 Gobezie, R., Kho, A., Krastins, B., Sarracino, D. A., Thornhill, T. S., Chase, M., Millett, P. J., \& 592 Lee, D. M. (2007). High abundance synovial fluid proteome: Distinct profiles in health

593

594

595 596

597

598

599

600

601

602

603

604

605

606

607

608

609

610

611

612

613

614

615

616 and osteoarthritis. Arthritis Research and Therapy, 9(2), 1-15. https://doi.org/10.1186/ar2172

Goldring S. R., Goldring M. B. (2016) Changes in the osteochondral unit during osteoarthritis: Structure, function and cartilage bone crosstalk. Nature Reviews Rheumatology.

Guingamp, C., Gegout-Pottie, P., Philippe, L., Terlain, B., Netter, P., \& Gillet, P. (1997). Monoiodoacetate-induced experimental osteoarthritis: A dose-response study of loss of mobility, morphology, and biochemistry. Arthritis and Rheumatism. https://doi.org/10.1002/art.1780400917

Huskisson, E. C., Berry, H., Gishen, P., Jubb, R. W., \& Whitehead, J. (1995). Effects of antiinflammatory drugs on the progression of osteoarthritis of the knee. Journal of Rheumatology.

Jiang, L., Li, L., Geng, C., Gong, D., Jiang, L., Ishikawa, N., Kajima, K., \& Zhong, L. (2013). Monosodium iodoacetate induces apoptosis via the mitochondrial pathway involving ROS production and caspase activation in rat chondrocytes in vitro. Journal of Orthopaedic Research. https://doi.org/10.1002/jor.22250

Jones HW, Bailey R, Zhang Z, Dunne KA, Blake DR, Cox NL, Morris CJ, Winyard PG. 1998. Inactivation of antithrombin III in synovial fluid from patients with rheumatoid arthritis. Annals of the Rheumatic Diseases. DOI: 10.1136/ard.57.3.162.

Kuyinu, E. L., Narayanan, G., Nair, L. S., \& Laurencin, C. T. (2016). Animal models of osteoarthritis: classification, update, and measurement of outcomes. Journal of Orthopaedic Surgery and Research, 11(1), 19. https://doi.org/10.1186/s13018-016-03465

Lelovas, K. L. P. (2014). Useful animal models for the research of osteoarthritis. 263-271. https://doi.org/10.1007/s00590-013-1205-2 
617 Liao, W., Li, Z., Zhang, H., Li, J., Wang, K., \& Yang, Y. (2015). Proteomic analysis of synovial 618 fluid as an analytical tool to detect candidate biomarkers for knee osteoarthritis. 619 International Journal of Clinical and Experimental Pathology.

620 Liu, C.-C., Lee, H.-C., Peng, Y.-S., Tseng, A. H., Wu, J.-L., Tsai, W.-Y., Wong, C.-S., \& Su, L.621 J. (2019). Transcriptome Analysis Reveals Novel Genes Associated with Cartilage 622 623 Degeneration in Posttraumatic Osteoarthritis Progression. CARTILAGE. https://doi.org/10.1177/1947603519847744

624

Lu, Q. Y., Han, Q. H., Li, X., Li, Z. C., Pan, Y. T., Liu, L., \& Fu, Q. G. (2014). Analysis of 625 differentially expressed genes between rheumatoid arthritis and osteoarthritis based on 626 627 the gene co-expression network. Molecular Medicine Reports. https://doi.org/10.3892/mmr.2014.2166

628 629

630

631

632

633

634

635

636

637 638

639

640

641

642

Luo Q, Qin X, Qiu Y, Hou L, Yang N. 2018. The change of synovial fluid proteome in rabbit surgery-induced model of knee osteoarthritis. American Journal of Translational Research.

Man G. S., Mologhianu G. 2014. Osteoarthritis pathogenesis - a complex process that involves the entire joint. Journal of medicine and life.

Manderson, G. A., Martin, M., Önnerfjord, P., Saxne, T., Schmidtchen, A., Mollnes, T. E., Heinegård, D., \& Blom, A. M. (2009). Interactions of histidine-rich glycoprotein with immunoglobulins and proteins of the complement system. Molecular Immunology. https://doi.org/10.1016/j.molimm.2009.07.011

Mateos, J., Lourido, L., Fernández-puente, P., Calamia, V., Fernández-lópez, C., Oreiro, N., Ruiz-romero, C., \& Blanco, F. J. (2012). Differential protein profiling of synovial fluid from rheumatoid arthritis and osteoarthritis patients using LC - MALDI TOF / TOF is. Journal of Proteomics, 75(10), 2869-2878. https://doi.org/10.1016/j.jprot.2011.12.042

McCoy AM. 2015. Animal Models of Osteoarthritis: Comparisons and Key Considerations. Veterinary Pathology. DOI: 10.1177/0300985815588611. 
643 Mohan G, Perilli E, Kuliwaba JS, Humphries JM, Parkinson IH, Fazzalari NL. 2011. Application 644 of in vivo micro-computed tomography in the temporal characterisation of subchondral 645 bone architecture in a rat model of low-dose monosodium iodoacetate-induced 646 osteoarthritis. Arthritis Research and Therapy. DOI: 10.1186/ar3543.

647 Mustafy T, Londono I, Villemure I. 2018. Can the contralateral limb be used as a control during 648 649 the growing period in a rodent model? Medical Engineering and Physics. DOI:

650

651

652

653

654 655

656

657

658

659

660

661

662

663

664

665

666

667 10.1016/j.medengphy.2018.04.013.

Nagira K, Ikuta Y, Shinohara M, Sanada Y, Omoto T, Kanaya H, Nakasa T, Ishikawa M, Adachi N, Miyaki S, Lotz M. 2020. Histological scoring system for subchondral bone changes in murine models of joint aging and osteoarthritis. Scientific Reports. DOI: 10.1038/s41598020-66979-7.

Naveen, S. V., Ahmad, R. E., Hui, W. J., Suhaeb, A. M., Murali, M. R., Shanmugam, R., \& Kamarul, T. (2013). Histology, glycosaminoglycan level and cartilage stiffness in monoiodoacetate-induced osteoarthritis: Comparative analysis with anterior cruciate ligament transection in rat model and human osteoarthritis. International Journal of Medical Sciences. https://doi.org/10.7150/ijms.6964

Nguyen, L. T., Sharma, A. R., Chakraborty, C., Saibaba, B., Ahn, M. E., \& Lee, S. S. (2017). Review of prospects of biological fluid biomarkers in osteoarthritis. In International Journal of Molecular Sciences. https://doi.org/10.3390/ijms18030601

Nylund, R., Lemola, E., Hartwig, S., Lehr, S., Acheva, A., Jahns, J., Hildebrandt, G., \& Lindholm, C. (2014). Profiling of low molecular weight proteins in plasma from locally irradiated individuals. Journal of Radiation Research. https://doi.org/10.1093/jrr/rru007

Osborn, T. M., Verdrengh, M., Stossel, T. P., Tarkowski, A., \& Bokarewa, M. (2008). Research article Decreased levels of the gelsolin plasma isoform in patients with rheumatoid arthritis. 10(5), 1-9. https://doi.org/10.1186/ar2520 
668 Park, S., Hwang, I. K., Kim, S. Y., \& Lee, S. (2006). Characterization of plasma gelsolin as a 669 substrate for matrix metalloproteinases. 1192-1199.

670 https://doi.org/10.1002/pmic.200500402

671 Piskin, A., Gulbabar, M. Y., Tomak, Y., Gulman, B., Hokelek, M., Kerimoglu, S., Koksal, B., 672 Alic, T., \& Kabak, Y. B. (2007). Osteoarthritis models after anterior cruciate ligament 673 resection and medial meniscectomy in rats: A histological and immunohistochemical 674 study. Saudi Medical Journal.

675 Pitcher, T., Sousa-Valente, J., \& Malcangio, M. (2016). The monoiodoacetate model of 676 osteoarthritis pain in the mouse. Journal of Visualized Experiments. 677 https://doi.org/10.3791/53746

678 Pritzker KPH, Gay S, Jimenez SA, Ostergaard K, Pelletier JP, Revell K, Salter D, van den Berg 679 WB. 2006. Osteoarthritis cartilage histopathology: Grading and staging. Osteoarthritis 680 and Cartilage 14:13-29. DOI: 10.1016/j.joca.2005.07.014.

681

682

683

684

Quintero-Fabián, S., Arreola, R., Becerril-Villanueva, E., Torres-Romero, J. C., Arana-Argáez, V., Lara-Riegos, J., Ramírez-Camacho, M. A., \& Alvarez-Sánchez, M. E. (2019). Role of Matrix Metalloproteinases in Angiogenesis and Cancer. In Frontiers in Oncology. https://doi.org/10.3389/fonc.2019.01370

Rose, B. J., \& Kooyman, D. L. (2016). A Tale of Two Joints : The Role of Matrix Metalloproteases in Cartilage Biology. 2016. https://doi.org/10.1155/2016/4895050

687

688

689

690

691

692 693

Rutgers M, van Pelt MJP, Dhert WJA, Creemers LB, Saris DBF. 2010. Evaluation of histological scoring systems for tissue-engineered, repaired and osteoarthritic cartilage. Osteoarthritis and Cartilage. DOI: 10.1016/j.joca.2009.08.009.

Salter, D. M., Su, S. L., \& Lee, H. S. (2014). Epidemiology and genetics of osteoarthritis. In Journal of Medical Sciences (Taiwan). https://doi.org/10.4103/1011-4564.147251

Scotece, M., Koskinen-Kolasa, A., Moilanen, T., Moilanen, E., \& Vuolteenaho, K. (2018). Novel adipokine associated with osteoarthritis: retinol binding protein 4 is produced by 
694

695

696

697

698

699

700

701

702

703

704

705

706

707

708

709

710

711

712

713

714

715

716

717

718

719

720

cartilage and correlates withmatrix metalloproteinasesin osteoarthritis patients. Osteoarthritis and Cartilage. https://doi.org/10.1016/j.joca.2018.02.276

Silacci, P., Mazzolai, L., Gauci, C., Stergiopulos, N., Yin, H. L., \& Hayoz, D. (2004). Gelsolin superfamily proteins: Key regulators of cellular functions. In Cellular and Molecular Life Sciences. https://doi.org/10.1007/s00018-004-4225-6

Sipe, J. D. (1995). Acute-phase proteins in osteoarthritis. Seminars in Arthritis and Rheumatism. https://doi.org/10.1016/S0049-0172(95)80020-4

Stenberg, J., Rüetschi, U., Skiöldebrand, E., Kärrholm, J., \& Lindahl, A. (2013). Quantitative proteomics reveals regulatory differences in the chondrocyte secretome from human medial and lateral femoral condyles in osteoarthritic patients. Proteome Science. https://doi.org/10.1186/1477-5956-11-43

Takahashi, I., Matsuzaki, T., \& Hoso, M. (2017). Long-term histopathological developments in knee-joint components in a rat model of osteoarthritis induced by monosodium iodoacetate. Journal of Physical Therapy Science. https://doi.org/10.1589/jpts.29.590

Teeple, E., Jay, G. D., Elsaid, K. A., \& Fleming, B. C. (2013). Animal models of osteoarthritis: Challenges of model selection and analysis. In AAPS Journal. https://doi.org/10.1208/s12248-013-9454-X.

Ucar, H. I., Tok, M., Atalar, E., Dogan, O. F., Oc, M., Farsak, B., Guvener, M., Yilmaz, M., Dogan, R., Demircin, M., \& Pasaoglu, I. (2007). Predictive significance of plasma levels of interleukin-6 and high-sensitivity $\mathrm{C}$-reactive protein in atrial fibrillation after coronary artery bypass surgery. The Heart Surgery Forum. https://doi.org/10.1532/HSF98.20061175

Vignon, E., Bejui, J., Mathieu, P., Hartmann, J. D., Ville, G., Evreux, J. C., \& Descotes, J. (1987). Histological cartilage changes in a rabbit model of osteoarthritis. Journal of Rheumatology.

Wanner, J. P., Subbaiah, R., Skomorovska-prokvolit, Y., Shishani, Y., Boilard, E., Mohan, S., Gillespie, R., Miyagi, M., \& Gobezie, R. (2013). Proteomic profiling and functional 
721

722

723

724

725

726

727

728

729

730

731

732

733 characterization of early and late shoulder osteoarthritis. Arthritis Research \& Therapy, 15(6), 1. https://doi.org/10.1186/ar4369

World Health Organization. (2013). Chronic rheumatic conditions. In Chronic deseases and health promotion.

Xie, L., Lin, A. S. P., Kundu, K., Levenston, M. E., Murthy, N., \& Guldberg, R. E. (2012). Quantitative imaging of cartilage and bone morphology, reactive oxygen species, and vascularization in a rodent model of osteoarthritis. Arthritis and Rheumatism. https://doi.org/10.1002/art.34370

Yang Y, Li P, Zhu S, Bi R. 2020. Comparison of early-stage changes of osteoarthritis in cartilage and subchondral bone between two different rat models. PeerJ. DOI: 10.7717/peerj.8934. 


\section{Table $\mathbf{1}$ (on next page)}

OARSI histopathological grade of femur and tibia in the control, week 4, week 8 and week 12 for the surgically and chemically induced group. 


\begin{tabular}{|c|c|c|c|c|c|c|c|c|}
\hline & \multicolumn{2}{|c|}{ Control } & \multicolumn{2}{|c|}{ Week 4} & \multicolumn{2}{|c|}{ Week 8} & \multicolumn{2}{|c|}{ Week 12} \\
\hline & $\begin{array}{c}\text { ACLT } \\
\text { (median, } \\
\text { range) }\end{array}$ & $\begin{array}{c}\text { MIA } \\
\text { (median, } \\
\text { range) }\end{array}$ & $\begin{array}{c}\text { ACLT } \\
\text { (median, } \\
\text { range) }\end{array}$ & $\begin{array}{c}\text { MIA } \\
\text { (median, } \\
\text { range) }\end{array}$ & $\begin{array}{c}\text { ACLT } \\
\text { (median, } \\
\text { range) }\end{array}$ & $\begin{array}{c}\text { MIA } \\
\text { (median, } \\
\text { range) }\end{array}$ & $\begin{array}{c}\text { ACLT } \\
\text { (median, } \\
\text { range) }\end{array}$ & $\begin{array}{c}\text { MIA } \\
\text { (median, } \\
\text { range) }\end{array}$ \\
\hline Femur & 0 & 0 & $\begin{array}{c}0 \\
(0-1)\end{array}$ & $\begin{array}{c}0.5 \\
(0-1.5)\end{array}$ & $\begin{array}{c}1 \\
(0.5-2)\end{array}$ & $\begin{array}{c}1.5 \\
(1-1.5)\end{array}$ & $\begin{array}{c}1 \\
(0-2.5)\end{array}$ & $\begin{array}{c}4.25 \\
(2-5.5)\end{array}$ \\
\hline Tibia & 0 & 0 & $\begin{array}{c}0 \\
(0)\end{array}$ & $\begin{array}{c}1 \\
(0.5-1)\end{array}$ & $\begin{array}{c}0 \\
(0-1)\end{array}$ & $\begin{array}{c}3 \\
(3-5)\end{array}$ & $\begin{array}{c}2 \\
(1.5-2.0)\end{array}$ & $\begin{array}{c}5 \\
(4.5-5.5)\end{array}$ \\
\hline
\end{tabular}

1 


\section{Table 2 (on next page)}

List of proteins and their log normalized volume for control, week 4, week 8 and week 12 for both induction groups.

The results are represented as mean $( \pm S D)$. 
Surgically-induced group

Protein

Mean $( \pm \mathrm{SD})$

\begin{tabular}{lllll} 
& Control & Week 4 & Week 8 & Week 12 \\
\hline alpha-1-antiproteinase F precursor & $5.11( \pm 0.3)$ & $5.1( \pm 0.37)$ & $5.5( \pm 0.3)$ & $5.35( \pm 0.23)$ \\
\hline histidine-rich glycoprotein & $4.94( \pm 0.17)$ & $5.03( \pm 0.06)$ & $5.1( \pm 0.39)$ & $5.41( \pm 0.35)$ \\
\hline retinol-binding protein 4 & $5.26( \pm 0.18)$ & $5.49( \pm 0.12)$ & $5.12( \pm 0.35)$ & $5.38( \pm 0.42)$ \\
\hline beta-actin-like protein 2 isoform & $4.56( \pm 0.28)$ & $4.83( \pm 0.21)$ & $4.49( \pm 0.38)$ & $4.91( \pm 0.04)$ \\
X1 & & & & \\
\hline Full=Ig kappa-b4 chain C region & $6.19( \pm 0.41)$ & $6.35( \pm 0.23)$ & $6.22( \pm 0.07)$ & $6.38( \pm 0.09)$ \\
\hline Serotransferrin & $5.15( \pm 0.2)$ & $5.34( \pm 0.3)$ & $5.58( \pm 0.51)$ & $5.75(0.18)$ \\
\hline Gelsolin isoform & $4.94( \pm 0.17)$ & $5.03( \pm 0.05)$ & $5.1( \pm 0.39)$ & $5.41( \pm 0.35)$
\end{tabular}

Chemically-induced group

\begin{tabular}{lllll}
\hline Protein & \multicolumn{3}{c}{ Mean $( \pm$ SD $)$} \\
\cline { 2 - 5 } & Control & Week 4 & Week 8 & Week 12 \\
\hline Serpin peptidase inhibitor & $6.21( \pm 0.10)$ & $6.47( \pm 0.08)$ & $6.28 \pm(0.22)$ & $6.18( \pm 0.15)$ \\
\hline Haptoglobin precursor & $4.85( \pm 0.04)$ & $5.05( \pm 0.13)$ & $5.38( \pm 0.15)$ & $5.07( \pm 0.27)$ \\
\hline Apolipoprotein I-IV precursor & $4.94( \pm 0.05)$ & $5.45( \pm 0.20)$ & $5.35( \pm 0.13)$ & $5.21( \pm 0.11)$
\end{tabular}

1 


\section{Table 3 (on next page)}

Characterization of proteins according to biological group for surgically and chemically induced group. 
Surgically-induced group

\begin{tabular}{lc}
\hline Biological process & Percentage (\%) \\
\hline Cellular process & 27 \\
\hline Cellular Component Organization \& Biogenesis & 27 \\
\hline Localization & 27 \\
\hline Biological Regulation & 18
\end{tabular}

Chemically-induced group

\begin{tabular}{lc}
\hline Biological process & Percentage (\%) \\
\hline Response to Stimulus & 23 \\
\hline Immune System Response & 15 \\
\hline Biological Regulation & 15 \\
\hline Metabolic Process & 15 \\
\hline Cellular Component Organization \& Biogenesis & 8 \\
\hline Cellular Process & 8 \\
\hline Biological Adhesion & 8 \\
\hline Localization & 8
\end{tabular}




\section{Figure 1}

Definition of tissue compartment in subchondral bone.

White square dotted line, cancellous bone (Cn.B), yellow round dotted line, bone marrow (BM). Calculation of BV/TV: Bone volume (BV/TV, \%) $=($ Cn.B region $-\mathrm{BM}$ regions $) * / C n . B$ region $\times 100, *$ Cn. B region $-B M$ regions $=$ Tb. $B$ regions.

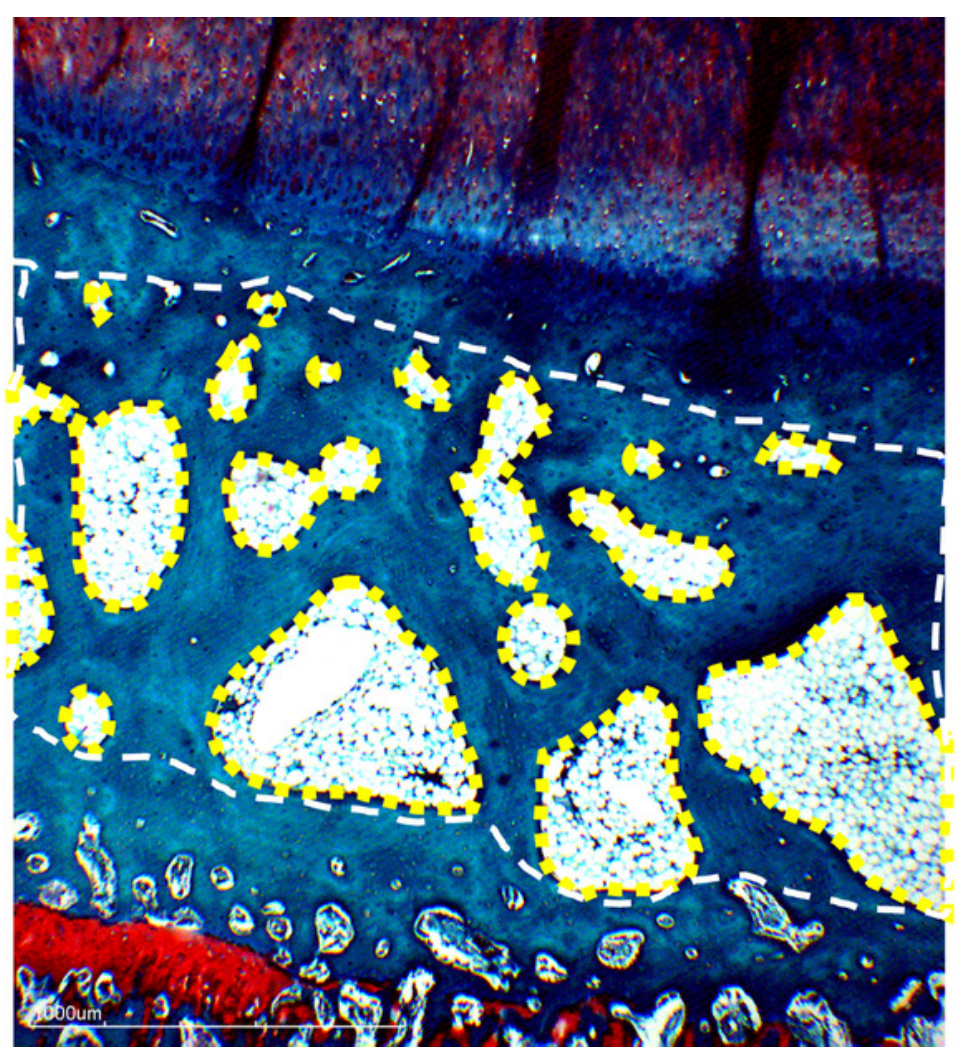

1. White square dotted line; cancellous bone (Cn.B)

2. Yellow round dotted line, bone marrow (BM)

Bone volume (BV/TV, \%)

$=\mathrm{Cn} \cdot \mathrm{B}-\mathrm{BM} / \mathrm{Cn} \cdot \mathrm{B} \times 100$

$=$ Trabecular bone $($ Tb.B) $/$ Cn.B x 100 
Figure 2

OARSI histopathological scores for (A) surgically induced femoral cartilage (B) chemically induced femoral cartilage (C) surgically induced tibial cartilage (D) chemically induced tibial cartilage.

Values are presented in median with $95 \%$ confidence interval $(n=5)$. Significant difference (Dunn's multiple comparisons, $p<0.05$ ) between column with the same letter within the groups.

(A)

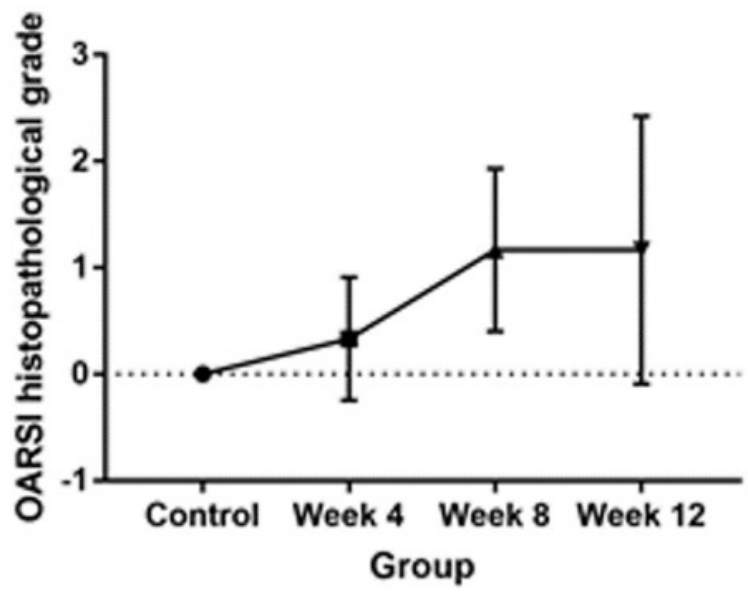

(C)

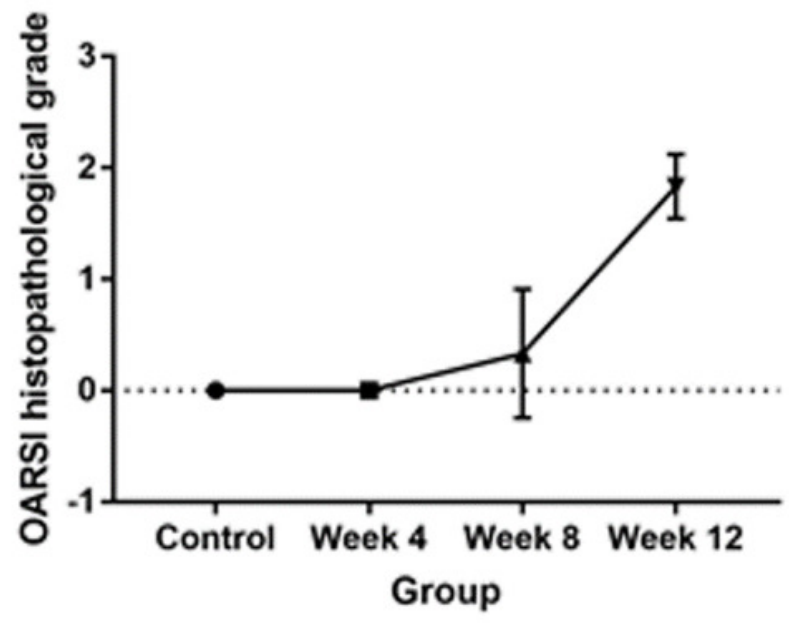

(B)

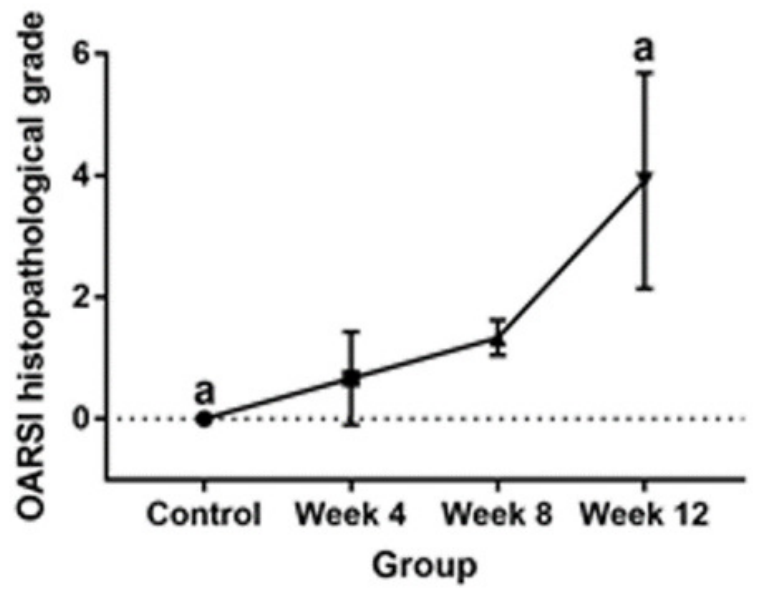

(D)

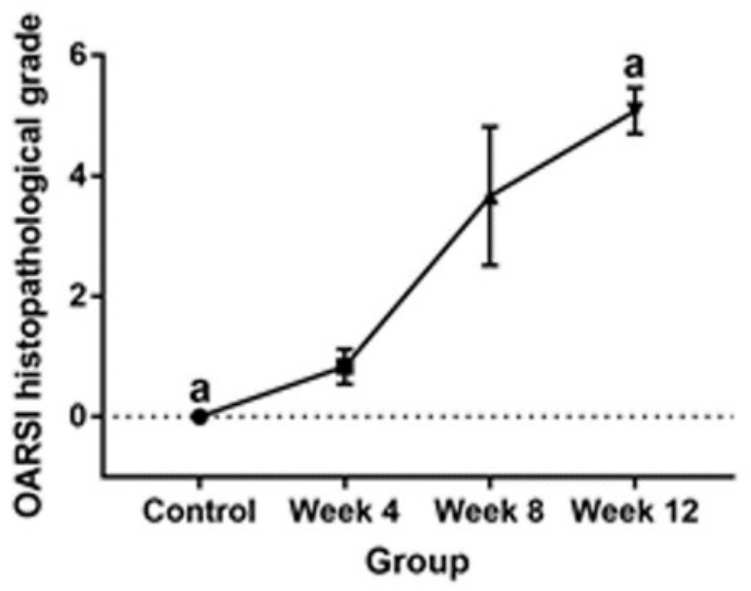




\section{Figure 3}

Representative histology images of femoral articular cartilage. A-D: surgically induced group. E-H: chemically induced group. Safranin-O/fast green stained with 20x magnification.

Surgically induced group showed apoptosis indicated by empty chondrons (red arrow) during week 4 and superficial fibrillation (red asterisk) during week 8. Chemically induced group showed surface erosion as early as week 8 (red arrowhead).
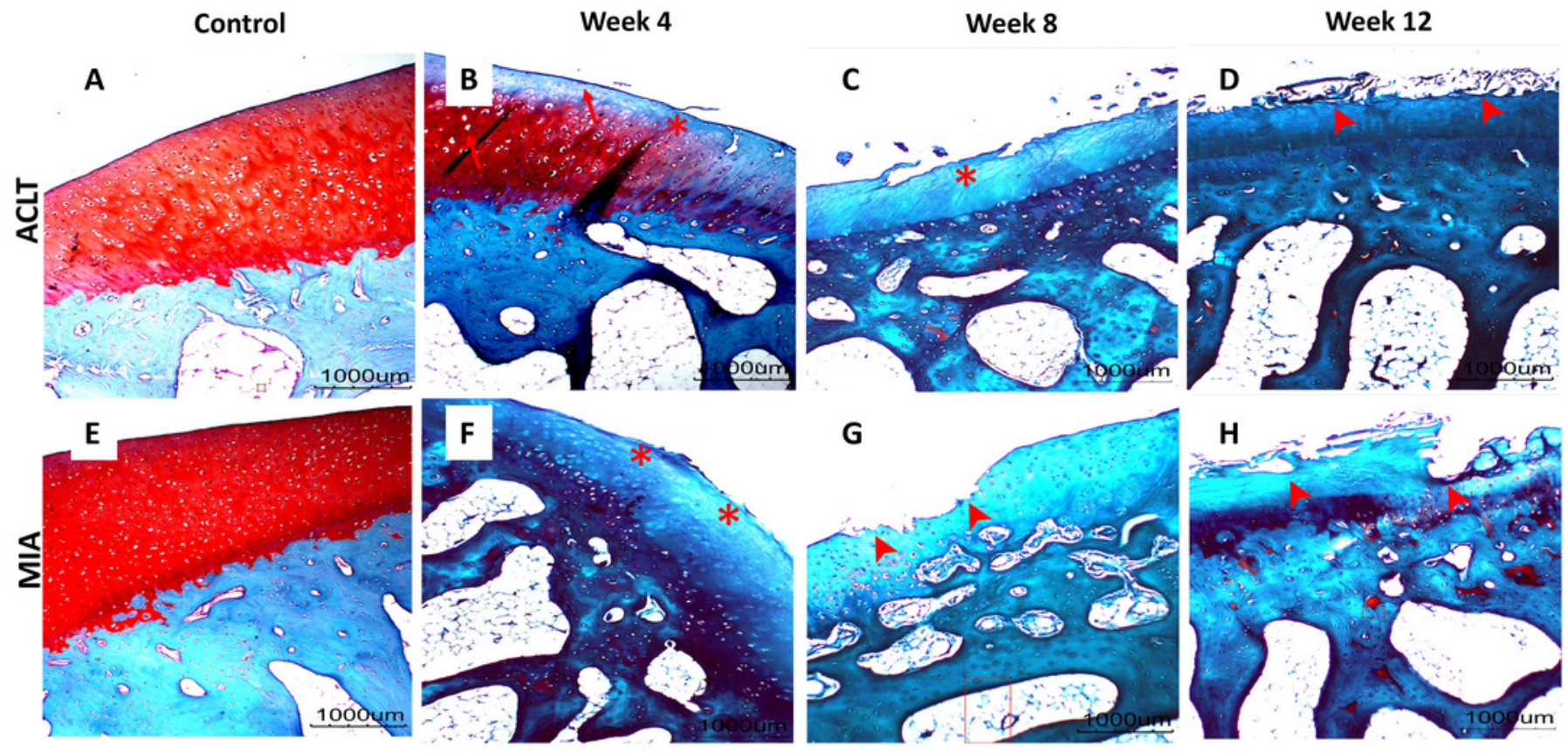


\section{Figure 4}

Representative histology images of tibial articular cartilage A-D: surgically induced group. E-H: chemically induced group. Safranin-O/fast green stained with 20x magnification.

During week 4, surgically induced group showed chondrocyte apoptosis (red arrow) and fibrillation (red asterisk) during week 8. Cartilage erosion (red arrowhead) were present during week 12. For chemically induced group, erosion can be observed during week 8 and week 12.
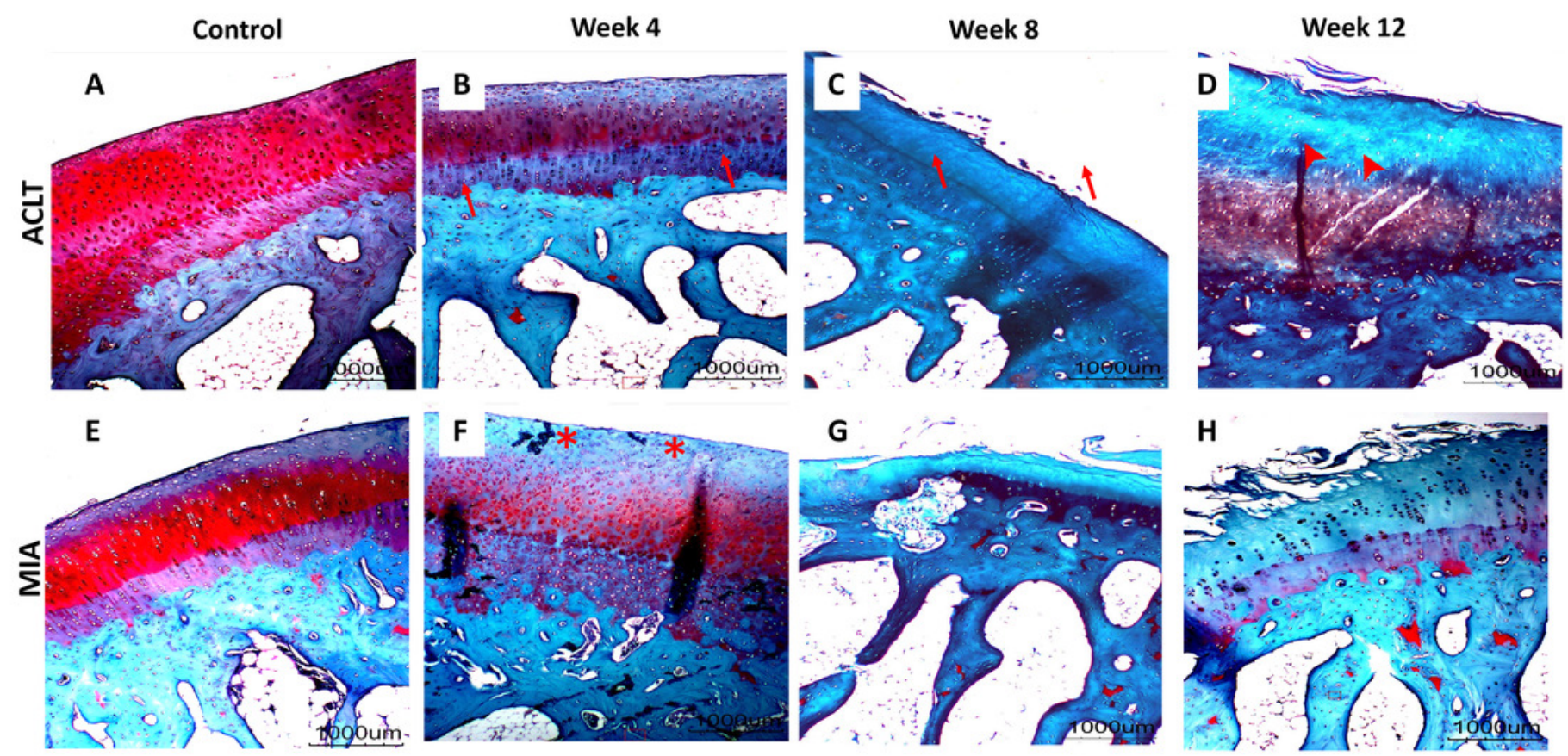
Figure 5

Subchondral bone BV/TV values for (A) femur and (B) tibia in week 4, week 8 and week 12 for the control, surgically (ACLT) and chemically (MIA) induced group.

No significant difference between groups were reported. The results are represented as mean $( \pm S D)$.

(A)

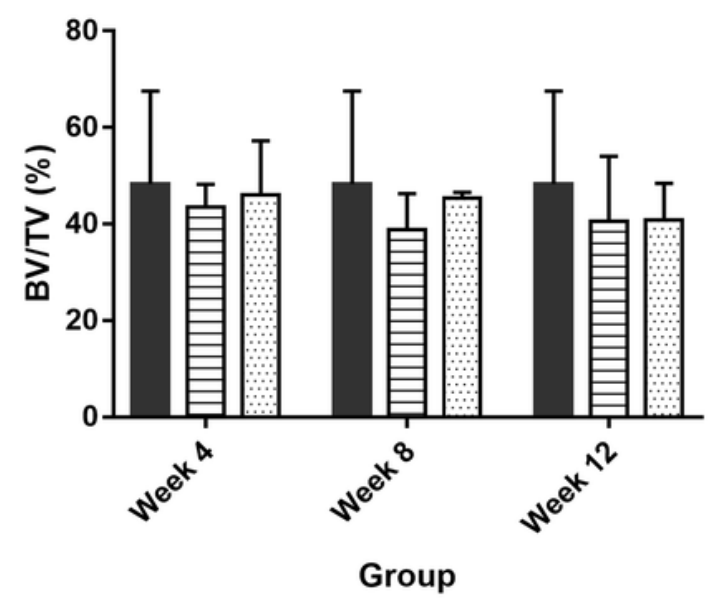

(B)

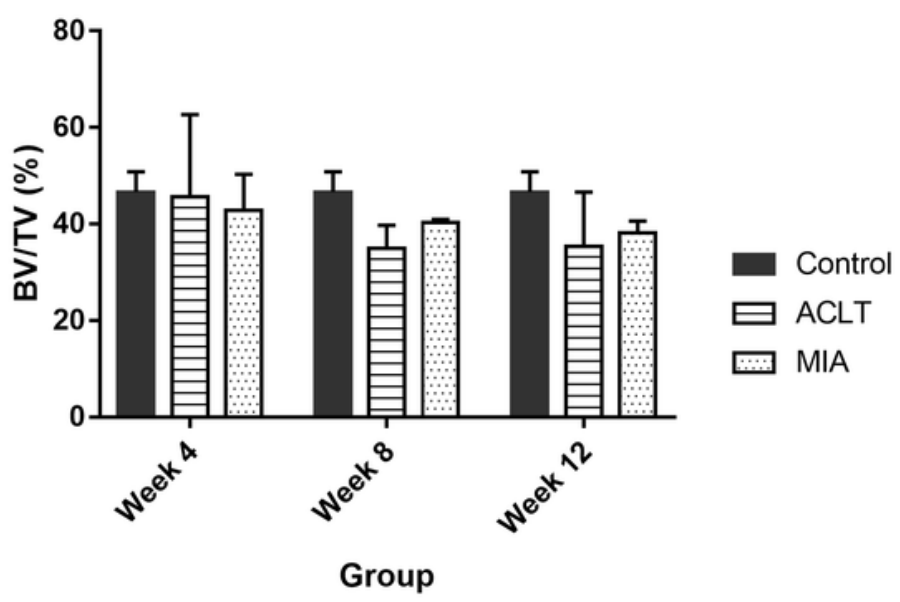




\section{Figure 6}

Representative 2DGE (pl 3-10) image for rabbit synovial fluid in (A) surgically induced group and (B) chemically induced group after staining with colloidal Coomassie stain.

Gels from respective groups were scanned and analyzed with Progenesis SameSpot software and protein spots (circled in red) were selected to be picked.
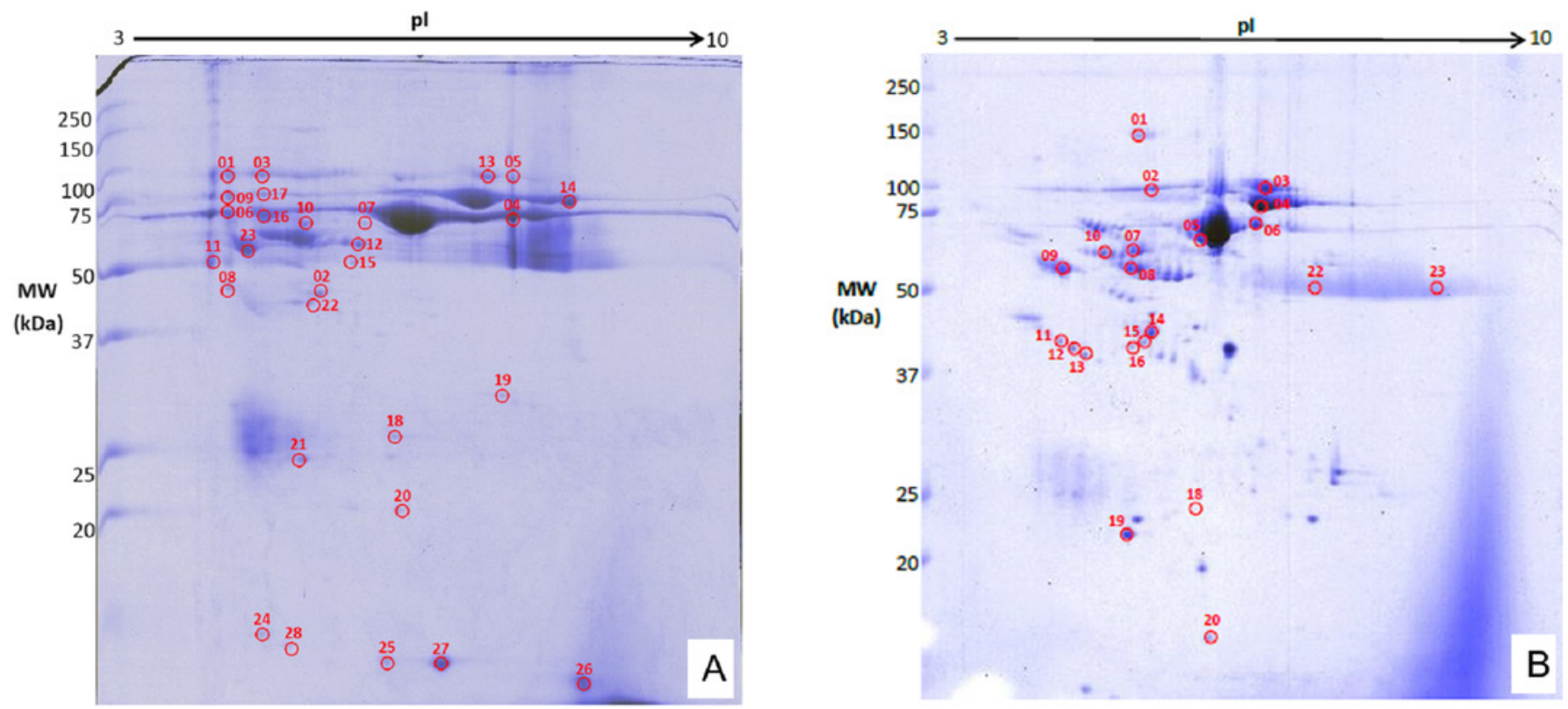